without a travel cost. They are cheap and easy to organise. Whilst the 'social' aspect of training is limited, this study suggests that webinars will remain an integral part of the postCovid era.

Conflict of Interest Nil

\section{ASSESSMENT OF A NOVEL INTEGRATED TRAINING PROGRAMME IN TRANTHORACIC ECHOCARDIOGRAPHY FOR JUNIOR CARDIAC PHYSIOLOGISTS IN A REGIONAL CARDIAC CENTRE}

Laurence O'Toole, Jane Mackay, Graham Fent, Oliver Watson. Sheffield Teaching Hospitals NHS Trust, Sheffield, UK

\subsection{6/heartjil-2021-BCS.80}

Introduction There is a national shortage of sonographers trained to British Society of Echo (BSE) transthoracic echocardiography (TTE) accreditation standard, which impacts directly on safe patient care. TTE training is arduous and impacts on departmental output, and $30 \%$ of echo departments in England had no cardiac physiologists training in TTE in 2018. In the face of a waiting list crisis due to loss of accredited staff, we introduced a novel, multifaceted and integrated TTE training programme for Agenda for Change (AfC) band 5 cardiac physiologists in January 2018. The aim was to train these much more junior staff, who were largely naïve to echo, to BSE accreditation standard at twice our previous rate.

Methods The training programme included (i) a foundation course with selection for further training on the basis of competence, (ii) an introductory scanning module, (iii) pathologyspecific training lists, (iv) training lists booked at reduced capacity (4 not 6 patients per list), (v) a complementary lecture programme, (vi) four levels of supervision with directions for both trainees and trainers of goals each week and (vii) ongoing review of progress. The primary end-point was the number of trainees per year achieving BSE accreditation standard compared to a historical control group (January 2010 to January 2018) in our hospitals. Secondary end-points include the impact of the training programme on departmental capacity and the duration of training.

Results In the control period, 7 sonographers were trained to BSE accreditation in a mean of 31 months (range 24 to 48 months), a training rate of 1.0 accredited sonographer/year. These staff were all AfC band 6, began training at an average age of 30 years, had a median of 4.7 years of prior postgraduate experience in cardiac physiology (range 0.75 to 5.25 years), and 4 had past echo training experience elsewhere. From January 2018 to Jan 2021, 11 physiologists entered the new training programme. These trainees were an average age of 25 years, median AfC Band of 5.0 and had a median of 1.5 years (range 0.25 to $11 \mathrm{yrs}$ ) of postgraduate experience in cardiac physiology. The training programme reduced departmental core echo capacity by $16 \%$ in the second year of implementation. Core capacity was recovered in the third year as trainees completed the programme. TTE out-patient waiting lists were maintained at less than 6 weeks by support from out-of-hours' work. Four trainees did not complete the programme. Five physiologists achieved full BSE accreditation by January 2021, in an average of 27 months of training (range 22 to 34 months) with some delays due to the 2020 Covid pandemic. This represents a training rate to independent scanning of 1.7 sonographers per year on the new programme.

Conclusion A programme of intensive training of echo-naïve very junior cardiac physiologists achieved a 70\% increase in the rate of attainment of BSE accreditation compared to historical performance with training completed in a similar timescale. This level of performance exceeds the need outlined in the 2015 strategic review of cardiac physiologist training. Conflict of Interest None

\section{ASSESSMENT OF A NOVEL, WEB-BASED, OPEN-ACCESS TRAINING RESOURCE FOR JUNIOR TRAINEES IN ECHOCARDIOGRAPHY}

Sally Chapman, Laurence O'Toole, Graham Fent, Jane Mackay. Sheffield Teaching Hospitals NHS Trust, Sheffield, UK

\subsection{6/heartjnl-2021-BCS.81}

Introduction Transthoracic Echocardiography (TTE) is a vital diagnostic tool in modern medicine. Training in TTE to the level of the national standard of accreditation required by the British Society of Echocardiography (BSE), which is necessary for independent practice, is arduous and teaching needs are often sacrificed to service demands. TTE training involves the acquisition of detailed anatomical, physiological and pathological knowledge of cardiac function in both health and disease as well as 2 to 3 years of hands-on experiential training. These demands have led to a severe shortfall of trained sonographers in the UK resulting in recurrent waiting list crises, which have an adverse impact on patient care. Web-based teaching is accessible, time efficient and economical and could be an effective adjunct to practical teaching in echocardiography. Currently, there is no free, open-access and comprehensive resource for trainee sonographers working to the British Society of Echocardiography curriculum.

Methods Funded by a grant from Health Education England, we have created a website to host the lectures, illustrative cases and demonstration videos used in the standardised training programme for TTE for junior sonographers in Sheffield Teaching Hospitals NHS Trust. The website [figure 1] is accessible on smartphones, tablets and PCs, and can be accessed at home and work. The content begins with basic concepts, moves to demonstrate aspects of image acquisition and optimisation, discusses the major cardiac pathologies and advises on image interpretation and reporting. Cardiac physiologists and cardiology registrars in our region were surveyed regarding their attitude to web-based echo learning and their impressions of the initial development of the website after development of 3 of the training modules.

Results Twenty-nine trainees responded to the initial survey with 18 (62\%) being cardiac physiologists. 90\% accessed online learning at least occasionally, most commonly on a weekly basis. The BSE website was their most frequently accessed website as an echo training resource. Of the respondents, 93\% reported that they would use an online echo training resource with $83 \%$ stating that using an online training resource would improve confidence in echo reporting. Over $90 \%$ felt that an online resource would improve their understanding of TTE. All of those who completed the follow up survey $(n=10)$ found the website helpful, said that they would continue use the site and would recommend the site to other trainees. 


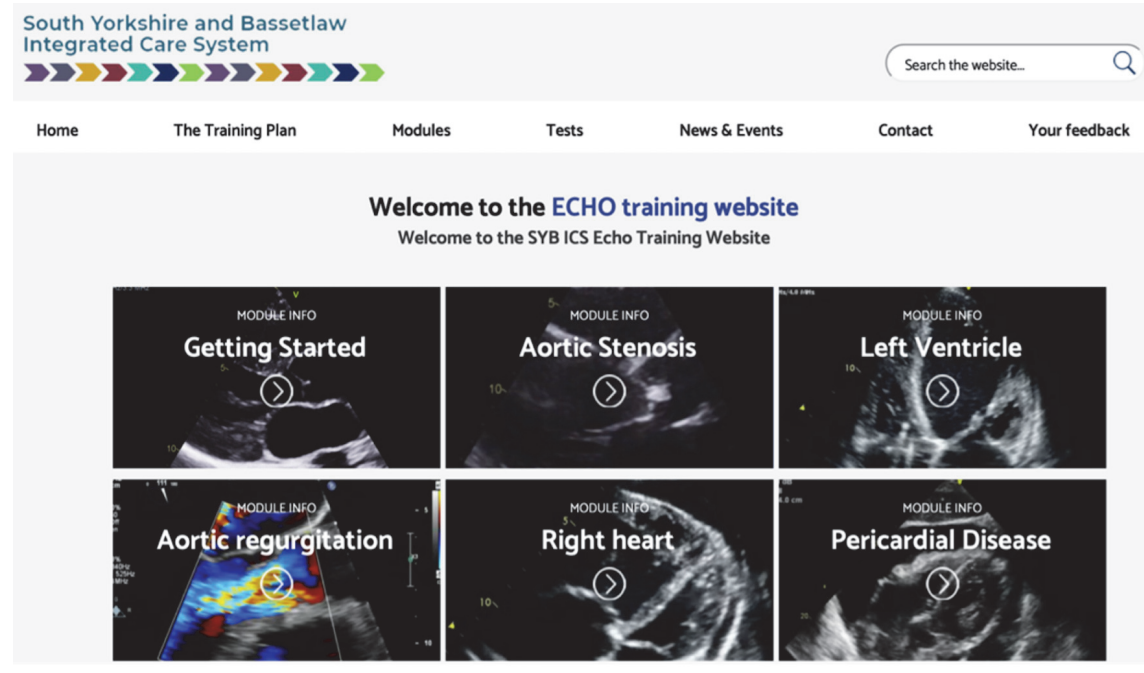

\section{Abstract 81 Figure 1}

Conclusion This training website seeks to provide a single source of access to the key knowledge needed for cardiac physiologists and registrars training in TTE. Our survey suggests that junior trainees will find it to be a useful adjunct to their experiential learning. The project is to continue to create content and we hope to open the website as a free, national open-access learning resource.

Conflict of Interest None

\section{\begin{tabular}{|l|l}
\hline 2 & OUT OF SIGHT BUT NOT OUT OF MIND. A DISTRICT
\end{tabular} HOSPITAL CARDIOLOGY TELECLINIC SETUP... PATIENTS', SUSTAINABILITY AND ENVIRONMENTAL BENEFITS}

${ }^{1}$ zhihong yao, ${ }^{2}$ Ahmed Farag, ${ }^{2}$ Pauline Mullineux, ${ }^{2}$ Wendy Matthews, ${ }^{2}$ Mark Carimichael, ${ }^{2}$ Chris Barlow. ' Scunthorpe General Hospital, Scunthorpe, UK; ${ }^{2}$ Warrington Hospital

\subsection{6/heartjnl-2021-BCS.82}

Introduction The ever-greater prevalence of cardiovascular disease has placed increasing pressure on cardiology services in the UK to achieve higher efficacy and improved quality of care. Telemedicine is emerging as a cost effective way to enhance patient's care. With the COVID19 pandemic, vitualconsultation had been became more widely used. The effectiveness and safety of tele-health intervention in cardiovascular condition in the UK remains unclear.

Aim To describe our early experience and outcomes setting up virtual teleconsultation for stable follow up cardiology patients in a district general hospital.

Method Since April 2019, a single cardiologist (AF) started enrolling selected stable cardiology follow up patients onto the virtual follow-up. Following the initial standard consultation, the results of the investigations would be reviewed by the attending cardiologist and subsequent consultation would be carried out via pre-arranged telephone appointment if patients are agreeable. Patient's outcomes and satisfaction survey were collated, sustainability and social benefits and environmental impact of the pilot were analysed.

Result A total of 116 patients were enrolled into the pilot. Fifty eight percent were males with an average age of 67 years (range 26-93). Conditions enrolled were: stable ischaemic heart disease (put numbers and percentage 42\%), Stable valvular heart disease (26\%), arrhythmias (13\%), heart failure (9\%) and others (9\%).

Sixty patients (52\%) were discharged and 42\% (49) patients had further routine tele-clinic follow-up. There were $6 \%$ (7) patients failed to attend the telephone consultation. Patients feedback was overwhelmingly positive with the main themes being: Convenience, avoiding car parking congestion, avoiding traffic / transportation issues as well as avoiding overall visit costs. The main concerns raised in patients using tele-consultation were related: 1) Mobile phone connectivity 2) Privacy issues and 3) Logistic of completing pre-requisite investigation prior appointment. Figure 1 demonstrates the clinic's sustainability benefits. The did not attend (DNA) rates were significantly less compared to standard clinic $(5.8 \%$ vs $8.2 \%)$. This pilot saved the trust c.a $£ 3000$ on consumables and logistics support and maximised outpatient clinic capacity utilisation.

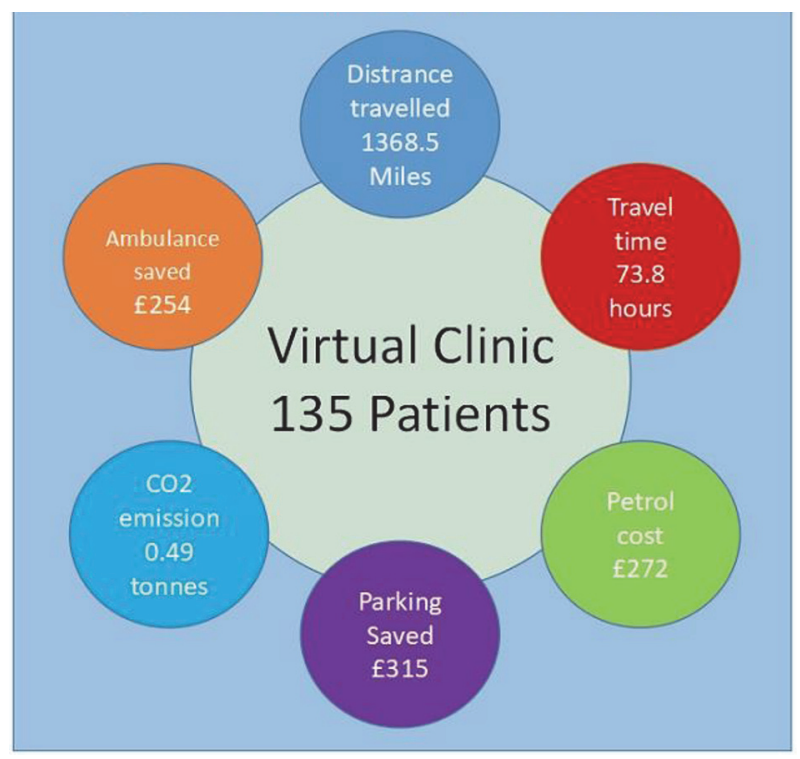

Abstract 82 Figure 1 The sustainbility benefits of virtual clinic 